\title{
NueVo Registro de BEgonia INERMIS (BegoniaceAe) PARA LA Flora ARgentina Y UN NUEVO SINÓNIMO DE B. FISCHERI
}

\author{
New RECord of Begonia INERMIS (BegoniaceAe) to the ARgentinian \\ FLORA AND A NEW SYNONYM OF B. FISCHERI
}

\author{
Christian A. Zanotti ${ }^{1 *}$ D, Ludovic J. C. Kollmann² ${ }^{\text {DD }}$ y Héctor A. Keller ${ }^{3}$ D
}

\begin{abstract}
1. Instituto de Limnología “Dr. Raú A. Ringuelet" (CONICET-FCNyM, UNLP), Bv. 120 y 62, 1900 La Plata, Buenos Aires, Argentina.

2. Museu de Biologia Prof. Mello Leitão, INMA, 296500-000, Santa Teresa, Espírito Santo, Brasil.

3. Instituto de Botánica del Nordeste, UNNE-CONICET, Casilla de Correo 209, 3400, Corrientes, Argentina.
\end{abstract}

*czanotti1979@gmail.com

\section{Citar este artículo}

ZANOTTI, C. A., L. J. C. KOLLMANN Y H. A. KELLER. 2020. Nuevo registro de Begonia inermis (Begoniaceae) para la Flora Argentina y un nuevo sinónimo de B. fischeri. Bol. Soc. Argent. Bot. 55: 471-478.

DOI: https://doi. org/10.31055/1851.2372.v55. n3.28429

Recibido: 30 Abril 2020

Aceptado: 7 Agosto 2020

Publicado: 30 Septiembre 2020

Editor: Diego Gutiérrez (DD

ISSN versión impresa 0373-580X

ISSN versión on-line 1851-2372

\section{SUMmARY}

Background and aims: The floristic richness of the province of Misiones (Argentina) has been increased in recent years, since new species have been cited and described for the region. The aims of this study were to revalidate and record Begonia inermis Imrsch. (Begoniaceae) for the Argentinian flora and propose a new synonym, B. hassleri C. DC., for B. fischeri Schrank.

M\&M: The new material collected and the new synonym were compared with the protologues and original materials of each species (except the original material of $B$. fischeri that could not be located), and additional specimens were also studied. The morphology was examined using optical microscopy. In addition, floras, taxonomic treatments and updated bibliography for the genus were reviewed.

Results and conclusions: The description of the new record is presented, a map with its geographical distribution is added, and field photographs and a key to identify the species of Begonia L. that inhabit Misiones are provided. The number of Begonia species cited for the Argentinian Flora was not modified, since $B$. inermis is revalidate and also we treat $B$. hassleri as a synonym of $B$. fischeri. The ring of trichomes at the apex of the petiole of $B$. inermis differentiates this species from the other species of Begonia that inhabit in Argentina.

\section{KEY WORDS}

Argentinian flora, Begonia fischeri, Begonia hassleri, Begonia inermis, Misiones, taxonomy.

\section{RESUMEN}

Introducción y objetivos: La riqueza florística de la provincia de Misiones se ha visto incrementada en los últimos años ya que se han citado y descripto nuevas especies para la región. Los objetivos de este trabajo fueron revalidar y registrar a Begonia inermis Imrsch. (Begoniaceae) para la flora de Argentina (Misiones) y proponer un nuevo sinónimo, B. hassleri C. DC., para B. fischeri Schrank..

M\&M: El nuevo material coleccionado y el nuevo sinónimo fueron comparados con los protólogos y materiales originales de cada especie (excepto el material original de $B$. fischeri que no pudo ser localizado), y, además, se estudiaron ejemplares adicionales. La morfología fue examinada utilizando microscopía óptica. Se revisaron floras, tratamientos taxonómicos y bibliografía actualizada para el género.

Resultados y conclusiones: Se presenta la descripción del nuevo registro, se agrega un mapa con su distribución geográfica actualizada, se proveen fotografías de campo y una clave para identificar las especies de Begonia L. que ocurren en Misiones. El número de especies de Begonia citadas para la Flora Argentina no se vio modificada, ya que se restituye y se cita a $B$. inermis y se sinonimiza a $B$. hassleri bajo $B$. fischeri. El anillo de tricomas en el ápice del pecíolo de $B$. inermis, diferencia a esta especie de las otras de Begonia que habitan en la Argentina.

\section{Palabras Clave}

Begonia fischeri, Begonia hassleri, Begonia inermis, Flora Argentina, Misiones, taxonomía. 


\section{INTRODUCCIÓN}

La provincia de Misiones es la que presenta la mayor riqueza florística de la República Argentina (Zuloaga et al., 1999, 2019) y este número se ha visto incrementado en los últimos años debido a que se han citado y descripto nuevas especies para la región, principalmente a causa de diversas exploraciones botánicas realizadas por el tercer autor de esta contribución (Keller, 2015a, b, 2017; Keller \& Crockett, 2015; Judkevich et al., 2016; Keller \& Tressens, 2016; Morillo et al., 2016; Zanotti \& Keller, 2017; Herrera et al., 2018; Marquez \& Keller, 2018; O’Leary \& Keller, 2018; Zanotti et al., 2018; Gatti \& Keller, 2019; Rojas \& Keller, 2019). Recientemente, se ha publicado un inventario actualizado de las plantas vasculares que crecen en la provincia incluyendo dichas novedades (Zanotti et al., 2020).

Begonia L. es el sexto género más grande de plantas vasculares con 1948 especies y presenta una distribución pantropical (Doorenbos et al., 1998; Shui et al., 2002; de Wilde \& Plana, 2003; Forrest \& Hollingsworth 2003; Neale et al., 2006; de Wilde, 2011; Hughes et al., 2015; Moonlight et al., 2018). A pesar de tener un probable origen africano, la mayor diversidad se concentra en Asia y en la región Neotropical (Goodall-Copestake et al., 2010), donde se halla en una gran variedad de hábitats, principalmente en bosques húmedos y sombríos. Sus especies se caracterizan morfológicamente por ser hierbas o subarbustos con tallos carnosos, estípulas vistosas, hojas frecuentemente con láminas asimétricas, flores unisexuales con tépalos, numerosos estambres centrípetos y un fruto proveniente de un ovario ínfero, frecuentemente 3-locular y generalmente 3-alado (Clement et al., 2004).

Colecciones recientes, efectuadas en la provincia Misiones, han permitido hallar un espécimen (Keller \& Rojas 13976, CTES, SI) perteneciente al género Begonia L., no citado hasta el momento para la Flora Argentina (Zuloaga et al., 2019) y que se ajusta a la descripción de $B$. inermis Irmsch. Esta especie ha sido tratada bajo la sinonimia de $B$. reniformis Dryand. por Smith \& Wasshausen (1983), criterio seguido por diversos autores en floras y tratamientos taxonómicos (i.e. Mamede et al., 2012), con excepción de Villada (2017) quien la considera como una entidad independiente de $B$. reniformis para la flora de Brasil en los estados de São Paulo y Paraná. En el presente trabajo, se revalida a $B$. inermis y se amplía el rango de su distribución para la Argentina (Misiones) y, por otro lado, procedemos a sinonimizar a $B$. hassleri C. DC. bajo B. fischeri Schrank, ya que no existen caracteres morfológicos discontinuos que permitan separar ambos taxones. Se provee una descripción morfológica, un mapa con la distribución geográfica, y fotografías de campo de la nueva cita y, además, se presenta una clave para diferenciar las siete especies de Begonia reconocidas para la provincia de Misiones.

\section{Materiales y Métodos}

El hallazgo de la nueva cita para la flora de Misiones, se fundamenta sobre la base de un ejemplar coleccionado en la localidad de Esperanza del departamento Iguazú por Keller \& Rojas 13976, coleccionado en diciembre del 2019, el cual ha sido depositado en el herbario CTES con su correspondiente duplicado en el herbario SI (acrónimos de acuerdo con Thiers, 2020). La nueva cita y la revalidación de Begonia inermis se basó de la comparación del material colectado con los materiales y descripciones originales de esta especie. Además, se estudiaron ejemplares adicionales para el sur de Brasil previamente citados por Villada (2017) para la complementación de la descripción de la especie. Para la sinonimización de $B$. hassleri bajo $B$. fischeri, se analizaron los protólogos y sólo se pudo acceder a examinar el material tipo de $B$. hassleri, ya que el de $B$. fischeri no fue posible localizarlo.

Las observaciones de los caracteres morfológicos fueron analizados bajo un microscopio estereoscópico (Zeizz, Stemi DV4). Para la realización de la clave dicotómica, se tomaron en cuenta las especies citadas para Misiones por Zanotti et al. (2020) y en base a los ejemplares y descripciones originales de cada uno de los taxones incluidos en la misma. Se examinaron los materiales de los siguientes herbarios: B, BM, CTES, G, M, MBM, MBML, SI, SP (Thiers, 2020).

\section{Resultados}

\section{Tratamiento taxonómico}

1. Begonia inermis Irmsch., Bot. Jahrb. Syst. 76: 39. 1953. TIPO: BRASIL. Estado de São 


\section{A. Zanotti et al. - Nuevo registro de Begonia inermis para Argentina}

Paulo. Beira do Rio Paranapanema, 4-XII-1899, A. Loefgren s.n. [Comissão Geográfica e Geológica de São Paulo 4429] (lectotipo, SP-8772 cb\#SP000048 [foto!], designado por Mamede et al., 2012: 102; isolectotipo, B cb\#10 0243028 [foto!]). Fig. 1A-D.

Hierbas terrestres o rupícolas de 0,7-1 m de alto, tallos con entrenudos de 5-14 cm de largo, erectos, glabros. Hojas pecioladas, basifijas, con pecíolo de 3-9,5 $\times 2 \mathrm{~mm}$, craso, glabro excepto por un anillo de tricomas simples, 1-celulares, de 1-2 mm de largo en el ápice, debajo de la inserción con la lámina; láminas largamente ovadas, de 7-20 × 6-12 cm, asimétricas, oblicuas o transversales, base cordada o lobulada, ápice agudo o acuminado, margen entero o aserrado, actinodrómas, membranáceas, glabras o glabrescentes con algunos tricomas simples, esparcidos en el margen y en el ápice de la cara adaxial; estípulas triangulares, de 7-10 $\times$ 4-5 mm, margen entero, escariosas, glabras, persistentes o caducas. Inflorescencias en cimas dicasiales plurifloras, de 17-20 cm de largo; flores numerosas, las estaminadas con 4 tépalos, blancos, los dos externos ovados, de 4-5 × 3-4 mm, margen entero, glabros y los dos internos oblongo-elípticos, de 3,5-4 × 1,5-2,5 mm, margen entero, glabros; estambres con los filamentos de 0,5-1 mm de largo, libres, y anteras lineares, de 1,5-2 mm de largo; las pistiladas con 5 tépalos, blancos, los dos exteriores elípticos, de 6-7 × 2-3 mm, margen entero, glabros y los tres interiores obovado-elípticos, de 3,5-8 $\times$ 2-4 mm, margen entero, glabros, bractéolas lineartriangulares, de 0,5-1,5 mm de largo, ápice agudo, glabras; gineceo con ovario elíptico, de 5-6,5 × 2,5$3 \mathrm{~mm}$, 3-locular, 3-alado, alas subiguales, la mayor de 2,7-3,5 mm de largo, ascendente, las dos menores de 1,2-2,1 mm de largo, glabro, estilos 3, cada uno dividido en dos ramas papilosas espiraladas, de 2,5-2,8 mm de largo. Fruto cápsula, ovado-oblonga, de 9-13 × 2-4 mm, base redondeada, ápice agudo, 3 -alado, alas desiguales, la mayor, triangular, de ca. $16 \times 8 \mathrm{~mm}$, ascendente, las dos menores de ca. $15 \times 5$ $\mathrm{mm}$, pedicelo fructífero de 5-7 $\mathrm{mm}$ de largo.

Distribución y hábitat. Especie citada para Brasil en los estados de São Paulo y Paraná, donde ha sido encontrada en el interior de los bosques estacionales semideciduos, en suelos rocosos o en madera en descomposición (Villada, 2017). En la Argentina se la encontró en Misiones (Fig. 2), en un bosque ripario con presencia de Euterpe edulis Mart. (Arecaceae); la población medra sobre basalto junto a una cascada sobre el curso de un arroyo pequeño, a escasa distancia del curso del río Paraná (Fig. 1E).

Observaciones taxonómicas. Begonia inermis fue sinonimizada bajo B. reniformis por Smith \& Wasshausen (1983) sin ninguna justificación que avale dicha acción taxonómica, y esta opinión fue seguida hasta la actualidad por otros autores (e.g. Mamede et al., 2012) con excepción de Villada (2017) que la mantiene como una especie independiente. Begonia reniformis es una especie bien representada en los herbarios brasileros y presenta una amplia variación morfológica en relación a las láminas foliares las cuales pueden ser enteras a profundamente lobuladas en sus márgenes, y las plantas pueden ser glabras o presentar diferentes grados de pilosidad, no presentando otra variabilidad morfológica significativa.

En el protólogo de la especie, Imrscher (1953: 39) describe como caracter diagnóstico la presencia de un anillo de tricomas en el ápice del pecíolo $\mathrm{y}$ alude que dicho anillo no es el mismo que se encuentra en B. neglecta A. DC., B. menbranacea A. DC., B. valdensium A. DC., B. longibracteata Brade y $B$. longibarbata Brade (este último indicado erróneamente como B. longibracteata Brade). Dichas especies poseen un anillo de tricomas de aproximadamente de $1 \mathrm{~cm}$ o más largos, diferentes a los que se hallan en $B$. inermis, que presentan tricomas de 1-2 mm de largo.

Cabe destacar que Begonia reniformis difiere principalmente de $B$. inermis por el porte menor de la planta que oscila en un rango de $30-40 \mathrm{~cm}$ versus $0,7-1 \mathrm{~m}$ de alto, por presentar la base de los tallos leñosos versus herbáceos $\mathrm{y}$, por último, por la ausencia del anillo de tricomas en el ápice del peciolo versus presencia del anillo, respectivamente. Por todos estos motivos, y luego de analizar los protólogos y los materiales tipo de $B$. inermis y $B$. reniformis (Banks \& Solander s.n., BM 001008546), concluimos que éstos dos taxones deben nuevamente ser consideradas como especies independientes. Por lo tanto, $B$. inermis es revalidada y citada en esta contribución para la flora de Argentina de acuerdo con lo expuesto por Villada (2017).

Otro aspecto a tener en cuenta es la confusión entre $B$. inermis y $B$. perdusenii Brade, ya que fueron encontrados numerosos ejemplares de la 
Bol. Soc. Argent. Bot. 55 (3) 2020
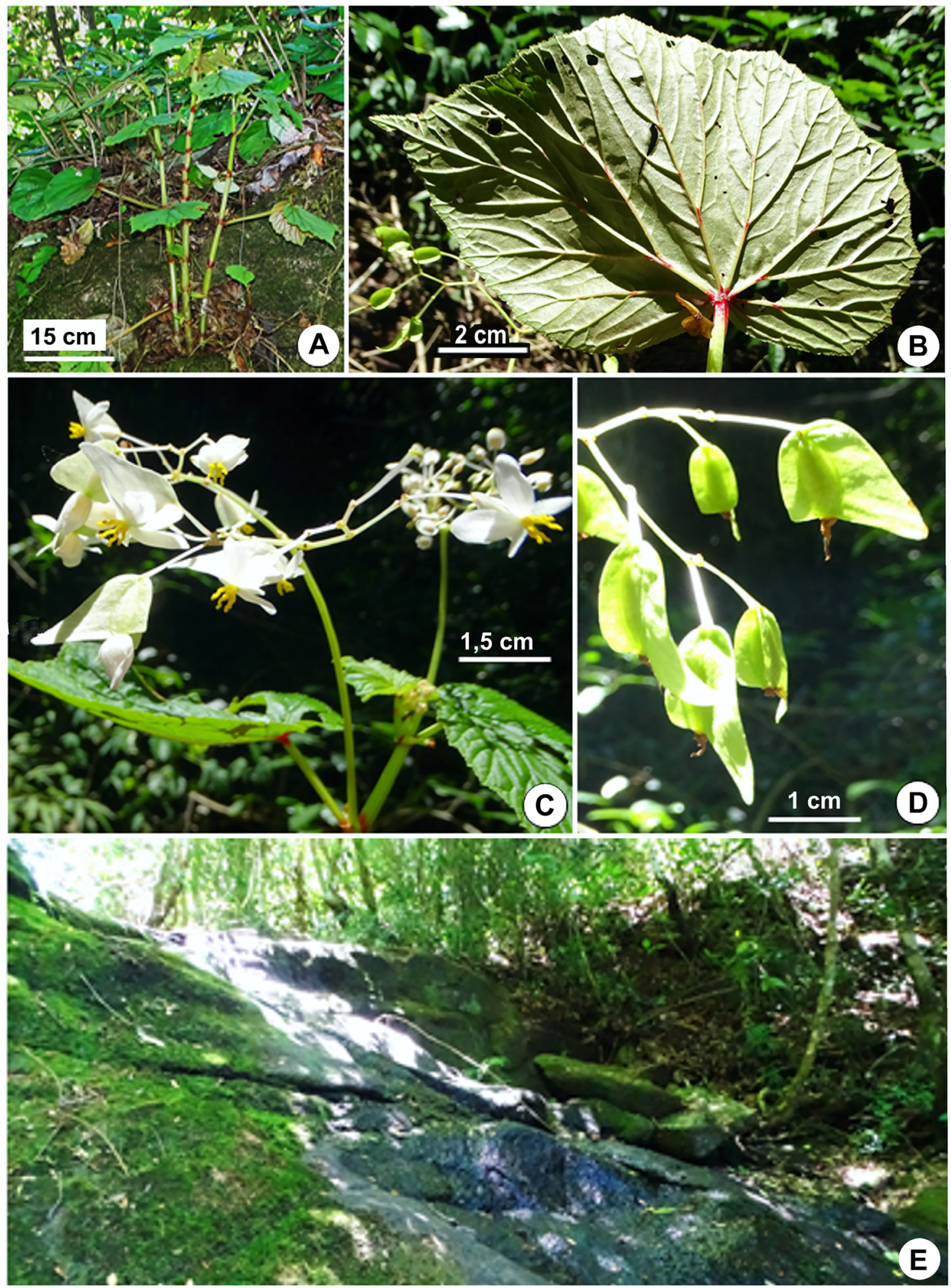

Fig. 1. Begonia inermis. A: Planta. B: Cara abaxial de la lámina foliar. C: Flores. D: Frutos. E: Ambiente. Fotos: H. A. Keller 


\section{A. Zanotti et al. - Nuevo registro de Begonia inermis para Argentina}

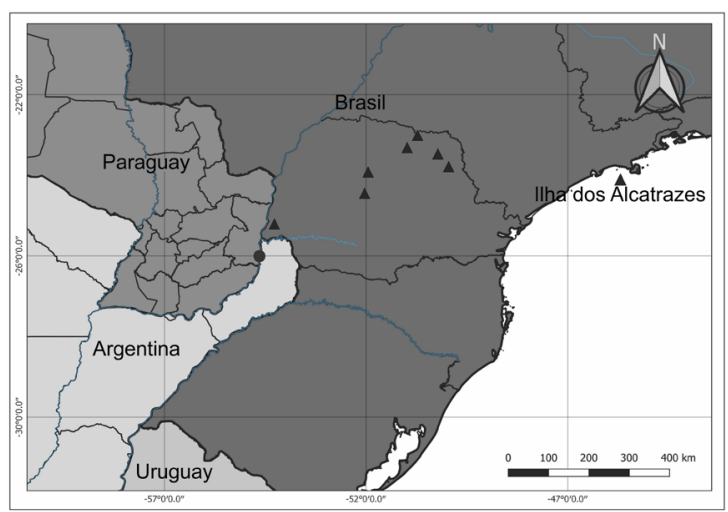

Fig. 2. Mapa de distribución de Begonia inermis. Círculo: distribución para la Argentina, triángulos: distribución para el Brasil.

primera especie erróneamente identificados bajo la segunda, la cual no presenta el característico anillo de tricomas en el ápice del pecíolo. Además, esta última especie presenta un indumento formado por tricomas glandulares y simples en toda la planta versus plantas totalmente glabras con la excepción del anillo de tricomas en el ápice del pecíolo y por la presencia de algunos pocos tricomas simples esparcidos en el margen y el ápice de la cara adaxial de la lámina en $B$. inermis. Por otro lado, la inflorescencia de $B$. perdusenii mide ca. $10 \mathrm{~cm}$ de largo versus ca. $20 \mathrm{~cm}$ de largo en $B$. inermis, y los tépalos externos de las flores pistiladas en $B$. perdusenii son pilosas versus totalmente glabras en B. inermis.

Material estudiado. ARGENTINA. Prov. Misiones: Depto. Iguazú, Esperanza, Puerto Carolina, $26^{\circ} 00^{\prime} 22,2^{\prime \prime} \mathrm{S} 54^{\circ} 40^{\prime} 32,8^{\prime \prime} \mathrm{W}, 7-X I I-$ 2019, Keller \& Rojas 13976 (CTES, SI). BRASIL. Estado de Paraná: Foz do Iguaçu, Rio Ocui, 7-XII1971, Hatschbach 23149 (MBM); Mun. Iretama, Rio Muquilão, 13-I-1983, Hatschbach 46005 (MBM); Mun. Ibiporã, Fazenda Doralice Ibiporã, 4-V-1999, Francisco et al. 3 (MBM); Mun. Jundiai do Sul, 9-II-2003, Carneiro 1408 (MBM); Mun. Fênix, Parque Estadual de Vila Rica do Espírito Santo, 25-XI-2000, Borgo 881 (MBM); Mun. Tomazina, Rio das Cinzas, 23-III-2007, Silva \& Barbosa 5632 (MBM). Estado de São Paulo: Mun. Santos, Santos, Ilha dos Alcatrazes, X-1920, Luederwldt \& Fonseca s.n. (B, SP).
2. Begonia fischeri Schrank, Pl. Rar. Hort. Monac. 2(6): tab 59. 1819 (1820), non Otto \& A. Dietr. (1936). TIPO: BRASIL. Estado de Rio de Janeiro, C.F.P. von Martius s.n. (no localizado).

Begonia hassleri C. DC., Bull. Soc. Bot. Genevé, sér 2, 8: 22, fig. 1. 1916. TIPO: ARGENTINA. Prov. Misiones, Depto. San Ignacio, X-1914, E. Hassler 460 (lectotipo, G cb\#00085120! [dos pliegos], designado por Delfini 2017: 9; isolectotipo, G cb\#00085121! [fragmento]), syn. nov.

Begonia fischeri es una especie altamente polimórfica, principalmente en relación a la forma, tamaño y grado de pilosidad de las láminas foliares. Se la caracteriza principalmente por presentar un indumento viloso blancuzco en los tallos, pecíolos y láminas foliares; esta última son generalmente cordiformes a largamente obovadas y las cápsulas presentan alas desiguales, siendo el ala mayor ascendente y aguda. La amplia variación morfológica se encuentra relacionada con su distribución geográfica (desde el sur de México hasta el noreste de la Argentina) y con los ambientes que ocupa, lo cual condujo a la descripción de diferentes variedades y especies que posteriormente fueron consideradas dentro de su sinonimia (Jacques \& Mamede, 2005; Delfini, 2017). El material original de Martius s.n. citado como tipo de B. fischeri en el protólogo, es posible que se encuentre destruido o que nunca se haya preparado. Sólo se ha podido localizar un ejemplar de Martius s.n. depositado en el herbario M (M0145725), pero no pudo ser confirmado que se trate del material original. La litografía y la breve descripción brindada por Schrank en la publicación original, coinciden con el material y descripción original de $B$. hassleri.

Begonia hassleri ha sido reconocida como una especie independiente y endémica para la flora de Misiones por Smith \& Schubert (1941) y posteriormente por Delfini (2017). Los primeros autores aludieron que no habían visto material de la especie y, por ende, sólo se remitieron a lo publicado en el protólogo brindado por De Candolle. Asimismo, Delfini, además de citar el material tipo depositado en $\mathrm{G}$, cita como material complementario examinado un ejemplar coleccionado en San Ignacio (Misiones) por Quiroga s.n. extraído de la página web del herbario MO (https://www.tropicos.org/home). Por este motivo, ha sido citado como uno de los 47 taxones endémicos registrados para la provincia de Misiones y clasificado como DD (datos deficientes) 
según los criterios de la IUCN para evaluar su estado de conservación (Zanotti et al., 2020).

En el protólogo de $B$. hassleri, el autor no realiza ninguna mención acerca de la relación con otras especies afines del género, sólo remarca que es un taxón interesante por presentar las cápsulas provistas de pilosidad y que éste es un caracter que se encuentra en un número reducido en los representantes de este género. Por otro lado, Smith \& Schubert (1941) diferencian a esta especie de $B$. fischeri, por esta última presentar una menor densidad del indumento en los tallos y en las hojas y por las alas del ovario "muy desiguales entre sí". Por último, Delfini (2017) diferencia a $B$. hassleri de $B$. fischeri, principalmente por las características de las estípulas: caducas y ciliadas en $B$. hassleri versus persistentes y fimbriadas en $B$. fischeri. Sin embargo, la gran plasticidad de la densidad del indumento no es un carácter discontinuo que permita separar a estos dos taxones. Asimismo, Smith \& Schubert (1941) indican que B. hassleri presenta las alas de ovario "escasamente desiguales" (en comparación con $B$. fischeri) pero según la ilustración del protólogo y el material tipo de $B$. hassleri, las alas del ovario son semejantes a las de $B$. fischeri $\mathrm{y}$, por ende, se observan superposiciones no permitiendo discriminar a estos dos taxones. Por otro lado, las características morfológicas de las estípulas establecidas para diferenciar a estas dos entidades por Delfini (2017), no es correcta, ya que en ambas especies son triangulares, persistentes y con el margen ciliado. Por todas estas razones, se subordina a $B$. hassleri bajo B. fischeri en esta contribución.

Material estudiado. ARGENTINA. Prov. Misiones: Depto. San Ignacio, Reserva Osununú, 8-XI-2018, Keller \& Rojas 13734 (CTES); Depto. San Pedro, Reserva Esmeralda, 13-V-1999, Deginani et al. 1322 (SI); Depto. Guaraní, borde del sendero hacia arroyo Yabotí, 12-XI-2000, Múlgura et al. 2603 (SI). BRASIL. Estado de Bahía: Mun. Guaratinga, Corrego jacutinga, 23IV-2009, Kollmann 11570 (MBML). Estado de Espírito Santo: Mun. Afonso Claudio, Lajinha, 5-VIII-2001, Kollmann 4311 (MBML); Mun. Atílio Vivácqua, Moitão, 27-IV-2007, Kollmann 9557 (MBML). Estado de Minas Gerais: Mun. Araponga, serra do Brigadeiro, 17-II-2005, Kollmann 13040 (MBML). Estado de Rio de Janeiro: Mun. Macaé, Frade de Macaé, sitio Roncador, 11-III-2013, Kollmann 12612 (MBML).

Como resultado del trabajo en Misiones se reconocen siete especies: $B$. fruticosa A. DC., $B$. cucullata Willd., B. descoleana L.B. Sm. \& B.G. Schub., B. inermis Irmsch., B. fischeri Schrank, B. perdusenii Brade y B. subvillosa Klotzsch.

\section{Clave para diferenciar a las especies de Begonia que crecen en Misiones}

1. Láminas foliares con venación pinnada; frutos con todas las alas rudimentarias y semejantes entre sí. .... B. fruticosa

1'. Láminas foliares con venación actinódroma; frutos con todas las alas desarrolladas y diferentes entre sí. . 2

2. Láminas foliares rectas, base truncada o anchamente cuneada. 3

3. Láminas foliares de ápice obtuso o redondeado; estípulas persistentes, margen dentado-ciliada. ..... B. cucullata

3'. Láminas foliares de ápice agudo o acuminado; estípulas caducas, margen entero. ...... B. descoleana 2'. Láminas foliares oblicuas o transversales, base cordada o lobulada.

4. Pecíolos glabros, excepto un anillo de tricomas en el ápice del pecíolo. B. inermis

4'. Pecíolos hirsutos o viloso-tomentosos en toda su extensión.

5. Pecíolos y láminas foliares con indumento hirsuto. B. fischeri

5'. Pecíolos y láminas foliares con indumento viloso-tomentoso.

6. Pecíolos y láminas foliares con tricomas septados y glandulares, blancuzcos; tallo glabro.

B. perdusenii

6'. Pecíolos y láminas foliares con tricomas simples y no glandulares, ferrugíneos; tallo tomentoso. 


\section{A. Zanotti et al. - Nuevo registro de Begonia inermis para Argentina}

\section{Conclusiones}

En esta contribución, no se modifica el número de especies de Begonia L. citadas para la Flora Argentina en la actualidad, ya que se restituye y se cita a $B$. inermis y se sinonimiza a $B$. hassleri bajo B. fischeri. El rango de distribución de $B$. inermis se amplía para la flora de Argentina (Misiones), ya que esta especie ha sido citada previamente para el sur del Brasil. La presencia del anillo de tricomas en el ápice del pecíolo que se encuentra en $B$. inermis, confirma la importancia taxonómica del carácter dado que diferencia a esta especie de las restantes del género representadas en la Argentina.

\section{Contribución de los Autores}

HK colectó el material y confeccionó las láminas. LK y CZ identificaron el material colectado. LK consultó herbarios brasileños y aportó datos para el desarrollo del manuscrito. CZ escribió y desarrolló el manuscrito.

\section{Agradecimientos}

Se agradece al Ing. Hugo Reis de la empresa Pindo S. A., quien propició la campaña de colección en Misiones donde se halló el ejemplar de referencia examinado. A Alain Chautems (G) por el envío de las fotos del tipo de Begonia hassleri, y también se desea agradecer a los herbarios consultados, B, BM, CTES, M, MBM, MBML, SI, SP. Por último, un agradecimiento especial a los editores y revisores que ayudaron a mejorar considerablemente el manuscrito.

\section{Bibliografía}

CLEMENT, W. L., M. C. TEBBIT, L. L. FORREST, J. E. BLAIR, L. BROUILLET, T. ERIKSSON \& S. M. SWENSEN. 2004. Phylogenetic position and biogeography of Hillebrandia sandwicensis (Begoniaceae): a rare Hawaiian relict. Amer. J. Bot. 91: 905-917. https://doi.org/10.3732/ajb.91.6.905

DELFINI, C. 2017. Begoniaceae. En: ZULOAGA, F. O. \& M. J. BELGRANO (eds.), Flora Vascular de la República Argentina, Vol. 17, pp. 1-16. Sigma, Buenos Aires.
DE WILDE, J. J. F. E. 2011. Begoniaceae. En: KUBITZKI, K. (ed.), Families and Genera of Vascular Plants, Vol. 10, pp. 56-71. Springer, Berlin. https://doi.org/10.1007/978-3-642-14397-7_5

DE WILDE, J. J. F. E. \& V. PLANA. 2003. A new section of Begonia (Begoniaceae) from west central Africa. Edinburgh J. Bot. 60: 121-130. https://doi.org/10.1017/S0960428603000118

DOORENBOS, J., M. S. M. SOSEF \& J. J. F. E. WILDE. 1998. The sections of Begonia, including descriptions, keys and species lists (Studies in Begoniaceae VI). Wageningen Agric. Univ. Pap. 98: 1-266.

FORREST, L. L. \& P. M. HOLLINGSWORTH. 2003. A recircumscription of Begonia based on nuclear ribosomal sequences. Plant Syst. Evol. 241: 193-211. https://doi.org/10.1007/s00606-002-0033-y

GATTI, F. E. \& H. A. KELLER. 2019. Voyria aphylla (Gentianaceae), la primera eudicotiledónea micoheterótrofa registrada para la Flora Argentina. Bonplandia 28: 159-162. https://doi.org/10.30972/bon.2823856

GOODALL-COPESTAKE, W. P., D. J. HARRIS \& P. M. HOLLINGSWORTH. 2010. The origin of a megadiverse genus: dating Begonia (Begoniaceae) using alternative datasets, calibrations and relaxed clock methods. Bot. J. Linn. Soc. 159: 363-380. https://doi.org/10.1111/j.1095-8339.2009.00948.x

HERRERA, J., F. E. GATTI \& H. A. KELLER. 2018. Securidaca rivinifolia (Polygalaceae) en la Argentina. Paraguay Biodiversidad 5: 52-54.

HUGHES, M., P. W. MOONLIGHT, A. JARA-MUÑOZ, M. C. TEBBITT, H. P. WILSON \& M. PULLAN. 2015-actual. Begonia Resource Centre [online]. Disponible en: http://padme.rbge.org.uk/begonia/ [Acceso: 2 Ene 2020].

JACQUES, E. L. \& M. C. H. MAMEDE. 2005. Notas nomenclaturais em Begonia L. (Begoniaceae). Revista Brasil. Bot. 28: 579-588.

https://doi.org/10.1590/S0100-84042005000300014

JUDKEVICH, M. D., R. M. SALAS \& H. A. KELLER. 2016. Randia brevituba (Rubiaceae), a new species from the Southern Cone of America and comments on Randia armata. Syst. Bot. 41: 238-244. https://doi.org/10.1600/036364416X690642

KELLER, H. A. 2015a. Austrochthamalia teyucuarensis (Apocynaceae: Asclepiadoideae), una nueva especie endémica de Misiones, Argentina. Lilloa 52: 40-45.

KELLER, H. A. 2015b. Oxypetalum radinsii (Apocynaceae: Asclepiadoideae), una nueva especie 
endémica de Misiones, Argentina. Bonplandia 24: 51-56. https://doi.org/10.30972/bon.241209

KELLER, H. A. 2017. Aenigma, un género sudamericano de Apocynaceae (Asclepiadoideae, Asclepiadeae) con tricomas vibrátiles en la corola. Lilloa 54: 58-73.

KELLER, H. A. \& S. L. CROCKETT. 2015. Hypericum robsonii spec. nova sect. Trigynobrathys (Hypericaceae) from the Misiones province in Argentina. Phyton (Horn) 55: 17-29.

KELLER, H. A. \& S. G. TRESSENS. 2016. Hedeoma teyucuarensis (Lamiaceae), una nueva especie de Misiones, Argentina. Darwiniana, n.s. 4: 5-11. https://doi.org/10.14522/darwiniana.2016.41.692

MAMEDE, M. C., S. J. GOMES DA SILVA, E. LIMA JACQUES \& B. C. ARENQUE. 2012. Begoniaceae. En: WANDERLEY, M. G. L., S. E. MARTINS, R. P. ROMANINI, T. SANT'ANNA MELHEM, G. J. SHEPERD, A. M. GIULIETTI, J. R. PIRANI, M. KIRIZAWA, M. M. R. F. DE MELO, I. CORDEIRO, \& L. S. KINOSHITA (eds.), Flora Fanerogâmica do Estado de São Paulo, vol. 7. pp. 1-380. Instituto de Botânica, São Paulo.

MARQUEZ, G. J. \& H. A. KELLER. 2018. Primer registro del género Actinostachys (Schizaeaceae) para Argentina. Bol. Soc. Argent. Bot. 53: 459-463. https://doi.org/10.31055/1851.2372.v53.n3.21318

MOONLIGHT, P. W., W. H. ARDI, L. A. PADILLA, K. CHUNG, D. FULLER, D. GIRMANSYAH, R. HOLLANDS, A. JARA-MUÑOZ, R. KIEW, W. LEONG, Y. LIU, A. MAHARDIKA, L. D. K. MARASINGHE, M. O'CONNOR, C. PENG, Á. J. PÉREZ, T. PHUTTHAI, M. PULLAN, S. RAJBHANDARY, C. REYNEL, R. R. RUBITE, J. SANG, D. SCHERBERICH, Y. SHUI, M. C. TEBBITT, D. C. THOMAS, H. P. WILSON, N. H. ZAINI \& M. HUGHES. 2018. Dividing and conquering the fastest-growing genus: Towards a natural sectional classification of the mega-diverse genus Begonia (Begoniaceae). Taxon 67: 267-323. https://doi.org/10.12705/672.3

MORILlO, G., S. A. CÁCERES MORAL \& H. A. KELLER. 2016. Cristobalia, un nuevo género sudamericano de Gonolobinae (Apocynaceae, Asclepiadoideae, Asclepiadeae). Pittieria 40: 122143.

NEALE, S., W. GOODALL-COPESTAKE \& C. A. KIDNER. 2006. The evolution of diversity in Begonia. En: TEIXEIRA DA SILVA, J. A. (ed.), Floriculture, Ornamental and Plant Biotechnology. Vol. IV, pp. 606-611, Global Science books, Middlesex.
O'LEARY, N. \& H. A. KELLER. 2018. Primer registro de Citharexylum solanaceum (Verbenaceae) para la flora Argentina. Darwiniana, n.s. 6: 108-112. https://doi.org/10.14522/darwiniana.2018.61.790

ROJAS, J. L. \& H. A. KELLER. 2019. Styrax acuminatus (Styracaceae), nuevo registro para la Flora Argentina. Darwiniania, n.s. 7: 174-178. https://doi.org/10.14522/darwiniana.2019.71.818

SHUI, Y. M., C. I. PENG \& C. Y. WU. 2002. Synopsis of the Chinese species of Begonia (Begoniaceae), with a reappraisal of sectional delimitation. Bot. Bull. Acad. Sin. 43: 313-327.

SMITH, L. B. \& B. G. SCHUBERT. 1941. Revisión de las especies argentinas del género Begonia. Darwiniana 5: 78-117.

SMITH, L. B. \& D. C. WASSHAUSEN. 1983. Notes on Begoniaceae I. Phytologia 52: 441-451. https://doi.org/10.5962/bhl.part.6896

THIERS, B. 2020. Index Herbariorum: a global directory of public herbaria and associated staff. New York Botanical Garden's Virtual Herbarium [online]. Disponible en: <http://sweetgum.nybg.org/ih> [Acceso: 2 Enero 2020].

VILLADA, J. C. 2017. Sinopse taxonômica do gênero Begonia L. (Begoniaceae) para a região sul do Brasil. Tesis de Maestría. Universidade Federal de Santa Catarina, Brasil.

ZANOTTI, C. A. \& H. A. KELLER. 2017. Nuevo registro y novedades taxonómicas de Piper miquelianum (Piperaceae, secc. Ottonia) para la Flora Argentina. Bonplandia 26: 51-56. https://doi.org/10.30972/bon.2612077

ZANOTTI, C. A., J. A. RADINS \& H. A. KELLER. 2018. Dos nuevos registros de Habenaria (Orchidaceae) para la Flora Argentina. Bol. Soc. Argent. Bot. 53: 435-442.

https://doi.org/10.31055/1851.2372.v53.n3.21316

ZANOTTI, C. A., H. A. KELLER \& F. O. ZULOAGA. 2020. Biodiversidad de la flora vascular de la provincial de Misiones, Región Paranaense. Darwiniana, n.s. 8: 42-291.

https://doi.org/10.14522/darwiniana.2020.81.878

ZULOAGA, F. O., O. MORRONE \& D. RODRÍGUEZ. 1999. Análisis de la biodiversidad en plantas vasculares de la Argentina. Kurtziana 27: 17-167.

ZULOAGA, F. O., M. J. BELGRANO \& C. A. ZANOTTI. 2019. Actualización del catálogo de plantas vasculares del Cono Sur. Darwiniana, n.s. 7: 208-278.

https://doi.org/10.14522/darwiniana.2019.72.861 